\title{
INTERSECTION NUMBERS OF SPECIAL CYCLES
}

\author{
JÜRGEN ROHLFS AND JOACHIM SCHWERMER
}

\section{INTRODUCTION}

Let $G / \mathbb{Q}$ be a connected semisimple algebraic group defined over $\mathbb{Q}$, and let $\sigma, \tau$ be two $\mathbb{Q}$-rational automorphisms of finite order of $G$ which commute with each other. We assume that the group $G(\mathbb{R})$ of real points of $G$ is noncompact. Let $\Gamma$ be a torsionfree arithmetic subgroup of $G(\mathbb{Q})$ which is stable under the group $\langle\sigma, \tau\rangle$ generated by $\sigma$ and $\tau$. Then $\sigma$ and $\tau$ act on the space $X$ of maximal compact subgroups of $G(\mathbb{R})$ and on the locally symmetric space $X / \Gamma$ in a natural way.

If $G(\mu)$ denotes the reductive algebraic $\mathbb{Q}$-group of fixed points of $\mu=\sigma, \tau$ acting on $G$, then the corresponding symmetric space $X(\mu)$ is acted upon by the arithmetic group $\Gamma(\mu)$ of fixed points of $\mu$ acting on $\Gamma$. These give rise to nonempty closed immersed submanifolds $C(\sigma)=X(\sigma) / \Gamma(\sigma)$ and $C(\tau)=$ $X(\tau) / \Gamma(\tau)$ of $X / \Gamma$. They are called special cycles. We assume that $X / \Gamma, C(\sigma)$, and $C(\tau)$ are oriented and that the intersection $C(\sigma) \cap C(\tau)$ is compact. Then, if $\operatorname{dim} C(\sigma)+\operatorname{dim} C(\tau)=\operatorname{dim} X$, the intersection number

$$
[C(\sigma)][C(\tau)] \in \mathbb{Z}
$$

is defined. It is the purpose of this paper to give a quite general analysis of the intersections and intersection numbers of these special cycles. As one of the results we obtain under some mild restrictions an 'explicit' formula (Theorem 4.11) for the intersection number of a pair of such cycles. This expression only depends on arithmetic data involved in describing the geometry of the connected components of the intersection. We make comments on a generalisation of this explicit formula in $\S 5$. The generalisation requires adelic methods, and the results obtained in this paper can be viewed as the necessary work to be done at the archimedean place.

The special cycles considered include the Hirzebruch-Zagier cycles [12], the cycles of Kudla-Millson [16], the cycles which occur in recent work of Jacquet and Rallis [15] and many others as special cases [18-21, 26, 27]. Our initial motivation in 1986 for this study was the desire to understand in a general

Received by the editors January 23, 1992 and, in revised form, October 9, 1992.

1991 Mathematics Subject Classification. Primary 11F75; Secondary 55N45.

Supported by Deutsche Forschungsgemeinschaft. 
framework the geometric construction of nonvanishing cohomology classes for arithmetic groups done by Millson-Raghunathan [21] in specific cases. The main focus of our work is to obtain a computable formula for these intersection numbers. Of course, this formula implies new nonvanishing results.

We describe the content of this paper in more detail.

From the results in [26], the fixed point components of an automorphism $\mu$ of finite order on $X / \Gamma$ can be parametrized by the first nonabelian cohomology set $H^{1}(\langle\mu\rangle, \Gamma)$ attached to $\langle\mu\rangle$. Combining these two parametrizations in the cases $\sigma$ and $\tau$ we obtain in $\S 1$ a description of the components of the intersection $C(\sigma) \cap C(\tau)$ in terms of the kernel of the natural restriction map

$$
\operatorname{res}_{\sigma} \times \operatorname{res}_{\tau}: H^{1}(\langle\sigma, \tau\rangle, \Gamma) \rightarrow H^{1}(\langle\sigma\rangle, \Gamma) \times H^{1}(\langle\tau\rangle, \Gamma) .
$$

We note that, even if $X(\sigma)$ and $X(\tau)$ intersect in exactly one point, the geometric nature of the fixed point components in $C(\sigma) \cap C(\tau)$ may be quite complicated, for example, higher-dimensional components may occur.

In $\S 2$ it is shown that the orientability assumptions which are necessary to define $[C(\sigma)][C(\tau)]$ can be satisfied by passing from $\Gamma$ to a suitable congruence subgroup. This subgroup may be chosen such that the components of the intersection $C(\sigma) \cap C(\tau)$ are orientable. Note that the problem of orientability of the various fixed point components is a main focus of both [20] and [21], where the intersections of cycles defined by involutions are considered. Our result is a general solution to this problem replacing the case-by-case discussion given in specific cases in $[20,21]$. Section 3 contains a proof of the formula

$$
[C(\sigma)][C(\tau)]=\sum_{\gamma \in \operatorname{ker}^{2} \operatorname{res}_{\sigma} \times \operatorname{res}_{\tau}} e(\eta(\gamma))[F(\gamma)]
$$

where each component $F(\gamma)$ of $C(\sigma) \cap C(\tau)$ contributes the Euler number of the excess bundle $\eta(\gamma)$ over $F(\gamma)$ as one summand. In $\S 4$ first we show that this Euler number is proportional to a volume of $F(\gamma)$ and we determine the proportionality factor. This factor is essentially the Euler number of a homogeneous bundle determined by $\eta(\gamma)$ on the compact dual symmetric space associated to $F(\gamma)$. It follows an analysis of the signs of $e(\eta(\gamma))[F(\gamma)]$ corresponding to elements $\gamma$ which map to the same class in $H^{1}(\langle\sigma, \tau\rangle, G(\mathbb{R}))$. In 4.5-4.8 we give conditions under which the numbers $e(\eta(\gamma))[F(\gamma)]$ are all of the same sign. This is the case if the actions of $G(\mathbb{R}), G(\sigma)(\mathbb{R})$, and $G(\tau)(\mathbb{R})$ on their symmetric space $X, X(\sigma)$, and $X(\tau)$ are all orientation preserving. For short, this is called condition (Or). Finally we obtain as Theorem 4.11: If $X(\sigma)$ and $X(\tau)$ intersect in exactly one point with positive intersection number and condition (Or) is satisfied then there exists a $\langle\sigma, \tau\rangle$-stable arithmetic subgroup $\Gamma_{1}$ of finite index in $\Gamma$ such that

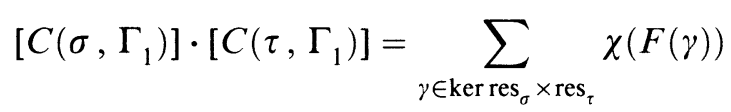


where all Euler characteristics $\chi(F(\gamma))$ are positive. Of course, then $[C(\sigma)]$ and $[C(\tau)]$ represent nontrivial classes in $H_{*}(\Gamma, \mathbb{C})$. Numerous classes of examples may be derived, for example, by use of the discussion in 4.8 where quite general conditions on $G$ and automorphisms of finite order are given which imply the assertion of (Or). Some of these are discussed in $\S 5$.

Notation. (i) Let $\Theta$ be a group acting on a set $M$. We write the action of $s \in \Theta$ on $m \in M$ as $m \mapsto s m={ }^{s} m=s(m)$ and put $M^{\boldsymbol{\Theta}}=M(\Theta)=\left\{\left.m \in M\right|^{s} m=\right.$ $m$ for all $s \in \Theta\}$. If $\Theta=\langle\sigma, \tau\rangle$ is generated by two elements $\sigma$ and $\tau$, we write $M(\Theta)=M(\sigma, \tau)$ and $M(\langle\sigma\rangle)=M(\sigma), M(\langle\tau\rangle)=M(\tau)$.

(ii) If the group $\Theta$ acts on a group $A$ as group of automorphisms, then $H^{1}(\Theta, A)$ denotes the first nonabelian cohomology set of this action. By definition, it consists of classes of cocycles, where a cocycle $\gamma$ is a map $\gamma: \Theta \rightarrow A$ written $s \mapsto \gamma_{s}$ with the property $\gamma_{s h}=\gamma_{s}{ }^{s} \gamma_{h}, h, s \in \Theta$. Cocycles $\gamma$ and $\xi$ are equivalent if there is an $a \in A$ such that $\xi_{s}=a^{-1} \gamma_{s}{ }^{s} a$ for all $s \in \Theta$. If $A$ and $\Theta$ act on a set $M$ in a compatible way, i.e., if $s(a m)={ }^{s} a^{s} m$ for all $s \in \Theta, a \in A, m \in M$ and if $\gamma$ is a cocycle for $H^{1}(\Theta, A)$ there is a $\gamma$-twisted $\Theta$-action on $M$ given by $m \mapsto \gamma_{s}{ }^{s} m$. We denote the fixed points of this action by $M(\gamma)$. If $\boldsymbol{\Theta}=\langle\sigma, \tau\rangle$ and if $\gamma=\left\{\gamma_{s}\right\}, s \in \boldsymbol{\Theta}$, is a cocycle as above, we denote by $\gamma_{\sigma}=\left\{\gamma_{t}\right\}, t \in\langle\sigma\rangle$, the cocycle for $H^{1}(\langle\sigma\rangle, A)$ given by the restriction of $\gamma$ to $\langle\sigma\rangle$. We write $M\left(\gamma_{\sigma}\right)$ for the fixed points of the $\gamma_{\sigma}$-twisted $\sigma$-action on $M$, similarly for the $\gamma_{\tau}$-twisted $\tau$-action. We get $M(\gamma)=M\left(\gamma_{\sigma}\right) \cap M\left(\gamma_{\tau}\right)$. To simplify the notation we put $H^{1}(\langle\sigma, \tau\rangle, A)=H^{1}(\sigma, \tau, A), H^{1}(\langle\sigma\rangle, A)=$ $H^{1}(\sigma, A)$, and $H^{1}(\langle\tau\rangle, A)=H^{1}(\tau, A)$. For further details on nonabelian cohomology we refer to [31, I, §5].

(iii) We give the usual meaning to the symbols $\mathbb{N}, \mathbb{Z}, \mathbb{Q}, \mathbb{R}, \mathbb{C}$. If $p$ is a prime of $\mathbb{Z}$, we denote the $p$-adic numbers by $\mathbb{Q}_{p}$.

(iv) For results and notation concerning algebraic groups we refer to [4].

\section{INTERSECTIONS OF SPECIAL CYCLES AND NONABELIAN COHOMOLOGY SETS}

In this section, $G$ is a connected reductive algebraic group defined over $\mathbb{Q}$.

1.1. Let $\sigma, \tau$ be two $\mathbb{Q}$-rational automorphisms of $G / \mathbb{Q}$ of finite order; we assume that $\sigma$ and $\tau$ commute with each other. Choose a maximal compact $\langle\sigma, \tau\rangle$-stable subgroup $K$ of $G(\mathbb{R})$ (cf. [10,13.5]). Then the group $\Theta:=\langle\sigma, \tau\rangle$ acts on $X=K \backslash G(\mathbb{R})$. Let $\Gamma$ be a $\Theta$-stable torsionfree arithmetic subgroup of $G(\mathbb{R})$. If $\gamma=\left(\gamma_{s}\right), s \in \Theta$, is a cocycle for $\Theta$ in $\Gamma$, we define a new $\gamma$-twisted $\Theta$ action on $G$ and on $\Gamma$ by $g \mapsto \gamma_{s}^{s} g \gamma_{s}^{-1}, g \in G, s \in \Theta$. The operation induced on $X$ is then given by $x \mapsto{ }^{s} x \gamma_{s}^{-1}, x \in X, s \in \Theta$. The new operation induced on $X / \Gamma$ coincides with the previous one. Let $\Gamma(\gamma)$ be the set of elements in $\Gamma$ fixed by the $\gamma$-twisted $\Theta$-action, and let $X(\gamma)$ be the fixed point set of the $\gamma$-twisted $\Theta$-action on $X$. Then the natural map $\pi_{\gamma}: X(\gamma) / \Gamma(\gamma) \rightarrow X / \Gamma$ is 
injective and its image

$$
F(\gamma)=\operatorname{im} \pi_{\gamma} \cong X(\gamma) / \Gamma(\gamma)
$$

lies in the fixed point set $\operatorname{Fix}(\Theta, X / \Gamma)$. We observe that $F(\gamma)$ only depends on the cohomology class in $H^{1}(\Theta, \Gamma)$ represented by the cocycle $\gamma$. Moreover, $F(\gamma)$ is nonempty since the action of $\Theta$ on $X$ is via isometries $[10, \mathrm{I}, 13.5]$, and $F(\gamma)$ is a closed immersed submanifold of $X / \Gamma$. We will now see that all fixed points of $\Theta$ arise by this construction. Consider a point $\bar{x} \in \operatorname{Fix}(\Theta, X / \Gamma)$ represented by $x \in X$. Then there exist uniquely determined elements $\gamma_{s} \in \Gamma$ such that ${ }^{s} x=x \gamma_{s}, s \in \boldsymbol{\Theta}$. One sees that $\gamma=\left(\gamma_{s}\right), s \in \boldsymbol{\Theta}$, is a cocycle for $H^{1}(\Theta, \Gamma)$. For another representative $y=x \cdot c, c \in \Gamma$, of $\bar{x}$ the attached cocycle is determined by $\gamma_{s}^{\prime}=c^{-1} \gamma_{s}{ }^{s} c, s \in \Theta$. Therefore every $\bar{x} \in \operatorname{Fix}(\Theta, X / \Gamma)$ determines uniquely a class in $H^{1}(\Theta, \Gamma)$, and the fixed point set is a disjoint union of the connected nonempty sets $F(\gamma), \gamma \in H^{1}(\Theta, \Gamma)$

$$
\operatorname{Fix}(\Theta, X / \Gamma)=\coprod_{\gamma \in H^{1}(\boldsymbol{\Theta}, \Gamma)} F(\gamma)
$$

If we consider the finite groups $\langle\mu\rangle, \mu=\sigma, \tau$, generated by $\sigma$ and $\tau$ respectively, these considerations apply as well. Thus we have

$$
\operatorname{Fix}(\mu, X / \Gamma)=\coprod_{\gamma \in H^{1}(\mu, \Gamma)} F(\gamma)
$$

as disjoint unions of connected nonempty sets. The connected component corresponding to the base point $1_{\mu}$ in $H^{1}(\mu, \Gamma)$ will be denoted by $C(\mu)=$ $C(\mu, \Gamma)$. It will be called a special cycle. Note that each of the components of $\operatorname{Fix}(\mu, X / \Gamma)$ may be viewed as a special cycle, for example, associated to the $\mathbb{Q}$-rational automorphism obtained by twisting $\mu$ with $\gamma$. If $G(\mu)$ denotes the reductive algebraic $\mathbb{Q}$-group of fixed points of $\mu$ acting on $G$ then $X(\mu):=X\left(1_{\mu}\right)$ is the symmetric space corresponding to $G(\mu)$, i.e., the inclusion $G(\mu)(\mathbb{R}) \rightarrow G(\mathbb{R})$ induces an isomorphism

$$
K(\mu) \backslash G(\mu)(\mathbb{R}) \stackrel{\sim}{\rightarrow} X(\mu) .
$$

It follows that the natural map

$$
X(\mu) / \Gamma(\mu) \stackrel{\sim}{\rightarrow} C(\mu)
$$

with $\Gamma(\mu):=\Gamma\left(1_{\mu}\right)=\{\gamma \in \Gamma \mid \mu(\gamma)=\gamma\}$ provides a diffeomorphism between the locally symmetric space $X(\mu) / \Gamma(\mu)$ and the closed immersed submanifold $C(\mu)$.

In general, a submanifold $C$ in some arithmetic quotient $X / \Gamma$ will be called a special cycle if $C=C(\sigma)$ for a suitable $\mathbb{Q}$-rational automorphism of $G / \mathbb{Q}$ of finite order. 
Now we give a parametrization of the connected components of the intersection of the two special cycles $C(\sigma)$ and $C(\tau)$. Recall that given a subgroup $\Xi$ of $\Theta$ there is natural restriction map res : $H^{1}(\Theta, \Gamma) \rightarrow H^{1}(\Xi, \Gamma),[31, I, \S 5]$.

Proposition 1.2. Let $\langle\sigma, \tau\rangle$ be the group generated by two rational $\mathbb{Q}$-automorphisms $\sigma, \tau$ of $G(\mathbb{Q})$ of finite order which commute with each other. Given a $\langle\sigma, \tau\rangle$-stable arithmetic subgroup $\Gamma$ of $G(\mathbb{Q})$ let

$$
\operatorname{res}_{\sigma} \times \operatorname{res}_{\tau}: H^{1}(\sigma, \tau, \Gamma) \rightarrow H^{1}(\sigma, \Gamma) \times H^{1}(\tau, \Gamma)
$$

be the map induced by the map which sends a cocycle $\gamma=\left(\gamma_{s}\right), s \in\langle\sigma, \tau\rangle$, to the pair of cocycles determined by $\left(\gamma_{s}\right), s \in\langle\sigma\rangle$, and $\left(\gamma_{t}\right), t \in\langle\tau\rangle$. Then we have:

(1) If $\gamma \in \operatorname{ker}\left(\operatorname{res}_{\sigma} \times \operatorname{res}_{\tau}\right)$ then $F(\gamma) \subset C(\sigma) \cap C(\tau)$ is a connected component of the intersection of $C(\sigma)$ and $C(\tau)$.

(2) There is a bijection

$$
\operatorname{ker}\left(\operatorname{res}_{\sigma} \times \operatorname{res}_{\tau}\right) \stackrel{\sim}{\rightarrow} \pi_{0}(C(\sigma) \cap C(\tau)),
$$

given by $\gamma \mapsto(F(\gamma))$, between the kernel of $\mathrm{res}_{\sigma} \times \mathrm{res}_{\tau}$ and the set of connected components of $C(\sigma) \cap C(\tau)$. The point determined by $F(\gamma)$ is denoted by $(F(\gamma))$.

Remark. By using the inf-res exact sequence (see [31, I-73/74]) one observes that the kernel of res $_{\sigma} \times$ res $_{\tau}$ is in bijection to $\operatorname{ker}\left(H^{1}(\sigma, \Gamma(\tau)) \rightarrow H^{1}(\sigma, \Gamma)\right)$ and $\operatorname{ker}\left(H^{1}(\tau, \Gamma(\sigma)) \rightarrow H^{1}(\tau, \Gamma)\right)$ as well.

Proof. Let $\gamma=\left(\gamma_{s}\right), s \in \Theta=\langle\sigma, \tau\rangle$, be a cocycle representing a class in $\operatorname{ker}\left(\right.$ res $_{\sigma} \times$ res $\left._{\tau}\right)$. The $\gamma$-twisted $\Theta$-action on $X$ is determined by the $\gamma_{\sigma}$-twisted $\sigma$-action and the $\gamma_{\tau}$-twisted $\tau$-action. Therefore, we have $X(\gamma)=X\left(\gamma_{\sigma}\right) \cap$ $X\left(\gamma_{\tau}\right)$. But the natural maps

$$
X(\gamma) \hookrightarrow X\left(\gamma_{\mu}\right) \hookrightarrow X \rightarrow X / \Gamma \quad, \quad \mu=\sigma, \tau,
$$

map $X(\gamma)$ to $C(\mu)$, whence $F(\gamma) \subset C(\sigma) \cap C(\tau)$. Conversely, if $p \in C(\sigma) \cap$ $C(\tau)$ then $p$ is an element of $\operatorname{Fix}(\Theta, X / \Gamma)$ since $\sigma$ and $\tau$ generate $\Theta$. By 1.1. (2) there is a unique element in $H^{1}(\Theta, \Gamma)$ represented by a cocycle $\gamma=$ $\left(\gamma_{s}\right), s \in \Theta$, such that $p \in F(\gamma)$. Since $C(\mu)$ corresponds to the base point $1_{\mu}$ in $H^{1}(\mu, \Gamma), \mu=\sigma, \tau$, the fact that $p \in C(\sigma) \cap C(\tau)$ holds implies that $\gamma_{\sigma}=$ $a^{-1 \sigma} a, \gamma_{\tau}=b^{-1 \tau} b$ for some $a, b \in \Gamma$. Thus, every point $p \in C(\sigma) \cap C(\tau)$ lies in a unique connected component $F(\gamma), \gamma \in \operatorname{ker}\left(\right.$ res $_{\sigma} \times$ res $\left._{\tau}\right)$; hence, assertions (1) and (2) hold.

1.3. Let $V$ be a $C^{\infty}$-manifold, and let $M$ and $N$ be two closed immersed submanifolds of $V$. Then we may view the tangent bundles $T M$ of $M$ and $T N$ of $N$ as subbundles of the tangent bundle $T V$ of $V$. We recall that $M$ and $N$ are said to intersect perfectly (or cleanly) if the connected components of the intersection $M \cap N$ are immersed submanifolds of $V$ and if for all such 
components $F$ of $M \cap N$ one has $T F=T M_{\mid F} \cap T N_{\mid F}$. Here the bundles are viewed as subbundles of the restriction $T V_{\mid F}$ of the tangent bundle $T V$ to $F$.

Retaining the notation and assumptions of Proposition 1.2 we have

Lemma 1.4. The special cycles $C(\sigma)$ and $C(\tau)$ in $X / \Gamma$ intersect perfectly.

Proof. Let the point $p \in C(\sigma) \cap C(\tau)$ be represented by $x \in X$. There is a unique element in $H^{1}(\Theta, \Gamma)$ represented by a cocycle $\gamma=\left(\gamma_{s}\right), s \in \Theta$, such that $p \in F(\gamma)$. Since the assertion as claimed is of local nature, it suffices to prove the corresponding statement for the intersection $X(\gamma)=X\left(\gamma_{\sigma}\right) \cap X\left(\gamma_{\tau}\right)$. But $\sigma$ and $\tau$ generate $\Theta$, and thus the claim follows from the fact that the exponential map exp : $T_{x} X \rightarrow X$ from the tangent space of $X$ at $x$ to $X$ is an equivariant bijection with respect to the $\gamma$-twisted $\Theta$-action.

Remark.1. (i) Millson and Raghunathan [21] consider the case that $\sigma$ and $\tau$ are involutions, and they assume that $X(\sigma) \times X(\tau) \stackrel{\sim}{\rightarrow} X$ holds where the map is induced by the obvious inclusions. Then $C(\sigma)$ and $C(\tau)$ have at least one isolated intersection point, where they intersect transversally. Given the involution $\sigma$ and a "rational" point $\bar{x} \in C(\sigma)$ represented by $x \in X(\sigma)$ one can take $\tau=\sigma \theta_{x}$ where $\theta_{x}$ is the Cartan involution corresponding to $x$. The rationality of $x$ here means that $\theta_{x}$ is an automorphism defined over $\mathbb{Q}$ of $G$. Instead of $\theta_{x}$ one also can use Cartan-like involutions (see [29] for this notion).

(ii) In this paper we work with automorphisms $\sigma$ and $\tau$ of finite order instead of involutions. This does not cause any essential complications. In order to show that this setting is meaningful we give a nonobvious example as follows.

Consider the quadratic form $q$ on $\mathbb{R}^{8}$ which is diagonalized with entries $(1,1,1,1,-1,-1,-1,-1)$. The corresponding special orthogonal group $G$ over $\mathbb{R}$ is denoted by $\mathrm{SO}(4,4)(\mathbb{R})$. Let $\sigma$ be the automorphism of $G$ which is given by conjugation with the diagonal matrix $\eta$ with entries $(1,1,1,1,1,1$, $-1,-1)$. Then $G(\sigma)(\mathbb{R})=\mathrm{S}(\mathrm{O}(4,2) \times \mathrm{O}(2))(\mathbb{R})$. The group $G$ contains a Cartan subgroup $T / \mathbb{R}$ such that $T(\mathbb{R})$ is compact and such that $T(\mathbb{R})=$ $\mathrm{SO}(2)(\mathbb{R}) \times \mathrm{SO}(2)(\mathbb{R}) \times \mathrm{SO}(2)(\mathbb{R}) \times \mathrm{SO}(2)(\mathbb{R})$ sitting blockwise on the diagonal. There is a diagram automorphism $\tau$ defined over $\mathbb{R}$ of order 3 on $G(\mathbb{C})$ such that $G(\tau)(\mathbb{R})$ is a split group of type $G_{2}$ and such that $\operatorname{SO}(2)(\mathbb{R}) \times 1 \times$ $1 \times 1 \times 1 \times \operatorname{SO}(2)(\mathbb{R})$ is a maximal compact torus of $G_{2}(\mathbb{R})$. Since $\eta$ lies in this torus, we get $\sigma \tau=\tau \sigma$. Let $X, X(\sigma), X(\tau)$ be as in 1.1. By inspecting [10, Chapter IX, $\S 4$, Table II] one sees that $\operatorname{dim} X(\sigma)=\operatorname{dim} X(\tau)=8$ and $\operatorname{dim} X=16$. Hence the assumptions of 3.4 hold for suitable subgroups $\Gamma$ of $\mathrm{SO}(4,4)(\mathbb{R})$.

\section{COVERINGS AND ORIENTABILITY}

In order to be able to define the intersection number of the special cycles $C(\sigma)$ and $C(\tau)$ as defined in $\S 1$ we need that the manifolds $X / \Gamma, C(\sigma)$, and $C(\tau)$ are orientable. For the actual determination of the intersection number we also need that all the connected components of the intersection $C(\sigma) \cap C(\tau)$ 
are orientable. In this section it is shown that a given arithmetic group $\Gamma$ contains a $\Theta$-stable normal arithmetic subgroup $\Gamma^{\prime}$ such that the special cycles determined by $\sigma$ and $\tau$ in $X / \Gamma^{\prime}$ and the connected components of their intersection are orientable.

In this section $G$ denotes an arbitrary linear algebraic group defined over $\mathbb{Q}$.

2.1. Let $\Gamma$ be an arithmetic subgroup of $G$; for any finite set $S$ of primes of $\mathbb{Q}$ we embed $\Gamma$ diagonally into $\prod_{p \in S} G\left(\mathbb{Q}_{p}\right)$. We recall that a subgroup $\Gamma^{\prime}$ of $\Gamma$ is called a congruence subgroup of $\Gamma$ if there are such a set $S$ and an open compact subgroup $U \subset \prod_{p \in S} G\left(\mathbb{Q}_{p}\right)$ such that $\Gamma^{\prime}=\Gamma \cap U$. Note that then $\Gamma^{\prime}$ is not necessarily an arithmetic congruence subgroup of $G(\mathbb{Q})$.

Proposition 2.2. Let $G / \mathbb{Q}$ be a linear algebraic group defined over $\mathbb{Q}$, and let $\Gamma \subset G(\mathbb{Q})$ be an arithmetic subgroup. Then there exists a congruence subgroup $\Gamma^{\prime}$ of $\Gamma$ such that $\Gamma^{\prime}$ is contained in the connected component of the identity $G(\mathbb{R})^{0}$ of the real Lie group $G(\mathbb{R})$.

Proof. Since the number of connected components of $G$ in the Zariski topology is finite, there exists an open compact subgroup $U_{p} \subset G\left(\mathbb{Q}_{p}\right), p$ a prime of $\mathbb{Q}$, such that $U_{p} \subset G^{0}\left(\mathbb{Q}_{p}\right)$. Then one has $\Gamma \cap U_{p} \subset G^{0}(\mathbb{Q})$. This reduces us to the case where $G$ is connected.

Let $G$ be connected and denote by $H$ the unipotent radical $R_{U} G$ of $G$. Then $D:=G / H$ is a connected and reductive linear group. The group $H(\mathbb{R})$ is connected. Therefore, we have a commutative diagram with exact rows and columns

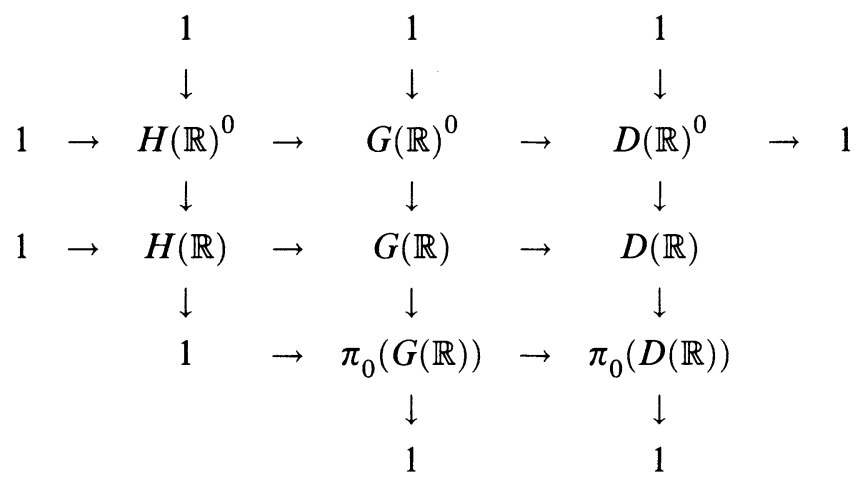

where $\pi_{0}()$ denotes the group of connected components.

We assume that our claim holds for connected reductive groups and show that then the claim holds for $G$. Indeed, if $\Gamma$ is arithmetic in $G$ then the image $\Gamma_{D}$ of $G$ in $D(\mathbb{R})$ under the natural projection $G \rightarrow D$ is arithmetic [1, 8.9]. By assumption there exists a finite set of primes $S$ and an open compact subgroup $W$ of $\prod_{p \in S} D\left(\mathbb{Q}_{p}\right)$ such that $W \cap \Gamma_{D} \subset D(\mathbb{R})^{0}$. We choose an open and compact subgroup $V$ of $\prod_{p \in S} G\left(\mathbb{Q}_{p}\right)$ which maps into $W$. Then the above diagram shows that $\Gamma \cap V \subset G(\mathbb{R})^{0}$. We have shown that it suffices to prove our claim for connected and reductive groups. 
We assume that $G$ is connected and reductive and that $\Gamma \subset G(\mathbb{Q})$ is arithmetic. Let $G_{\text {Der }}$ be the connected component of the derived group of $G$. Then $G_{\text {Der }}$ is semi-simple and there is a simply connected covering group denoted by $G_{\text {sc }}$ of $G_{\text {Der }}$ and a natural $\mathbb{Q}$-rational map $\varphi: G_{\text {sc }} \rightarrow G$ with image $G_{\text {Der }}$ and finite central kernel. Let $Z$ be the connected component of the center of $G$. Then $Z$ is a torus defined over $\mathbb{Q}$. We define a $\mathbb{Q}$-rational map

$$
f: G_{\mathrm{sc}} \times Z \rightarrow G
$$

such that $f(g, z)=\varphi(g) z^{2}$. Then $f$ is a group homomorphism where $\operatorname{ker} f$ is central and finite. Since $G$ is connected and $\operatorname{dim} Z+\operatorname{dim} G_{\text {sc }}=\operatorname{dim} G$, we see that $f$ is surjective (over $\overline{\mathbb{Q}}$ ). We abbreviate $\mu=\operatorname{ker}(f), G_{1}:=G_{\mathrm{sc}} \times Z$. From the exact sequence

$$
1 \rightarrow \mu(\mathbb{C}) \rightarrow G_{1}(\mathbb{C}) \rightarrow G(\mathbb{C}) \rightarrow 1
$$

we get the exact sequence

$$
1 \rightarrow \mu(\mathbb{R}) \rightarrow G_{1}(\mathbb{R}) \rightarrow G(\mathbb{R}) \stackrel{\partial_{\infty}}{\rightarrow} H^{1}(\mathbb{C} \mid \mathbb{R}, \mu(\mathbb{C}))
$$

where $\partial_{\infty}$ is defined as in [31, Chapter I, 5.4]. We have $f\left(G_{1}(\mathbb{R})\right)=G(\mathbb{R})^{0}$. To see this we use that $Z(\mathbb{R})=\prod \mathrm{SO}(2) \times \prod \mathbb{R}^{*}$ for a suitable number of copies of the factors and we use that $G_{\mathrm{sc}}(\mathbb{R})=G_{\mathrm{sc}}(\mathbb{R})^{0}$; see $[4,4.7]$. Hence $f\left(G_{1}(\mathbb{R})\right)$ is connected. On the other hand, $\operatorname{dim} f\left(G_{1}(\mathbb{R})\right)=\operatorname{dim} G(\mathbb{R})$ and $f\left(G_{1}(\mathbb{R})\right)=G(\mathbb{R})^{0}$ follows. In order to prove our claim we therefore have to find a congruence subgroup $\Gamma^{\prime}$ of $\Gamma$ such that $\partial_{\infty} \mid \Gamma^{\prime}=1$.

From the exact sequence

$$
1 \rightarrow \mu(\overline{\mathbb{Q}}) \rightarrow G_{1}(\overline{\mathbb{Q}}) \rightarrow G(\overline{\mathbb{Q}}) \rightarrow 1
$$

we get an exact sequence

$$
1 \rightarrow \mu(\mathbb{Q}) \rightarrow G_{1}(\mathbb{Q}) \stackrel{f}{\rightarrow} G(\mathbb{Q}) \stackrel{\partial}{\rightarrow} H^{1}(\overline{\mathbb{Q}} / \mathbb{Q}, \mu(\overline{\mathbb{Q}})) .
$$

Using $[1,8.9]$ we see that $f f^{-1}(\Gamma)$ is a subgroup of finite index in $\Gamma$. Therefore, $\partial(\Gamma)$ is a finite subgroup of $H^{1}(\overline{\mathbb{Q}} / \mathbb{Q}, \mu(\overline{\mathbb{Q}}))$. Thus there is a finite Galois extension $k \subset \overline{\mathbb{Q}} \subset \mathbb{C}$ of $\mathbb{Q}$ such that $\partial(\Gamma) \subset H^{1}(k / \mathbb{Q}, \mu(k))$. Because $\mu$ is finite, we may (and will) choose $k$ so that $\mu(k)=\mu(\bar{k})$ holds.

Let $S$ be a finite set of primes such that all primes $p \notin S$ are unramified in $k$ and such that $\mu, G_{1}, G$, and $f$ are defined over the ring $\mathbb{Z}_{S}$ of $S$-integral integers. Moreover, we choose $S$ so large that the exponent of $\mu(\overline{\mathbb{Q}})$ is prime to all $p \notin S$ and such that $\Gamma \subset G\left(\mathbb{Z}_{p}\right)$ for $p \notin S$. Let $k_{w}$ be the completion of $k$ with respect to a place $w$ of $k$ which extends $p \notin S$ : We denote by $\mathscr{O}_{w}$ the integers in $k_{w}$ and by $\mathfrak{p}_{w}$ the maximal ideal in $\mathscr{O}_{w}$. Then $\mu(k)=\mu\left(k_{w}\right)=$ $\mu\left(\mathscr{O}_{w}\right)$ and reduction $\bmod \mathfrak{p}_{w}$ induces a bijection $\mu\left(\mathscr{O}_{w}\right) \stackrel{\sim}{\rightarrow} \mu\left(\mathscr{O}_{w} / \mathfrak{p}_{w}\right)$. To see this we choose an algebraic closure $\overline{\mathbb{F}}_{p}$ of $\mathbb{Z} / p \mathbb{Z}=\mathbb{F}_{p}$ which contains $\mathscr{O}_{w} / \mathfrak{p}_{w}$. Now $\mu\left(\overline{\mathbb{F}}_{p}\right)$ is a product of cyclic groups, all of order prime to $p$. Therefore, 
by Hensels Lemma the natural map $\mu\left(\mathscr{O}_{w}\right) \rightarrow \mu\left(\overline{\mathbb{F}}_{p}\right)$ is bijective with image $\mu\left(\mathscr{O}_{w} / \mathfrak{p}_{w}\right)$.

The embedding $k \subset \overline{\mathbb{Q}} \subset \mathbb{C}$ determines an archimedean value $\infty$ of $k$. We get a commutative diagram

$$
\begin{array}{ccc}
\Gamma & \stackrel{\partial}{\rightarrow} H^{1}(k / \mathbb{Q}, \mu(k)) \stackrel{\text { res }}{\rightarrow} & H^{1}\left(k_{\infty} / \mathbb{R}, \mu\left(k_{\infty}\right)\right) \\
\downarrow & & \downarrow \\
G(\mathbb{R}) & \partial_{\infty} & H^{1}(\mathbb{C} / \mathbb{R}, \mu(\mathbb{C}))
\end{array}
$$

If $k$ is totally real then $k_{\infty}=\mathbb{R}$ and $\partial_{\infty} \mid \Gamma=1$, i.e., our claim holds. So let us assume that $k_{\infty}=\mathbb{C}$. We write $F_{\infty}$ for the automorphism of $k$ over $\mathbb{Q}$ which induces complex conjugation on $\mathbb{C}=k_{\infty}$.

If $w$ is a finite place of $k$ extending $p \notin S$ we write $F_{w}$ for the corresponding Frobenius automorphism of $k_{w}$ over $\mathbb{Q}_{p}$. The Frobenius automorphism over $\mathbb{Z} / p \mathbb{Z}=\mathbb{F}_{p}$ is denoted by $F_{p}$. Then we get a commutative diagram

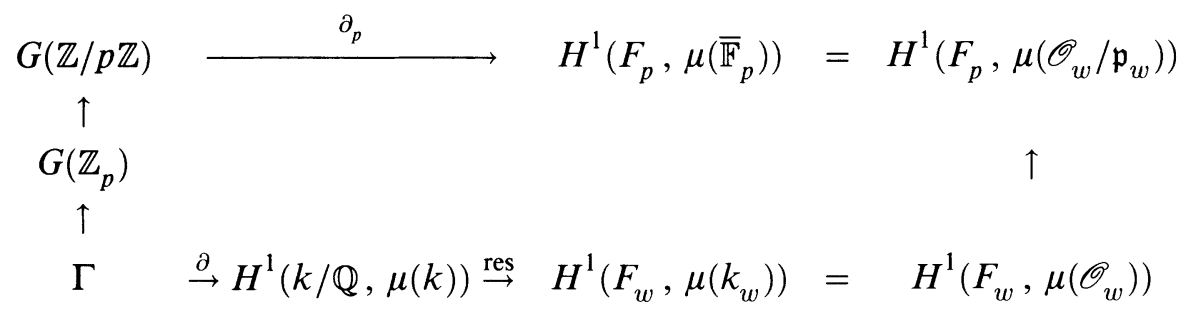

The right vertical arrow is induced by the isomorphism $\mu\left(\mathscr{O}_{w}\right) \stackrel{\sim}{\rightarrow} \mu\left(\mathscr{O}_{w} / \mathfrak{p}_{w}\right)$ and gives a bijection onto $H^{1}\left(\mathbb{F}_{q} / \mathbb{F}_{p}, \mu\left(\mathbb{F}_{q}\right)\right)$ where we put $\mathbb{F}_{q}=\mathscr{O}_{w} / \mathfrak{p}_{w}$. By the Tchebotarev density theorem [17, VIII, $\S 4$, Theorem 10] we can choose $w$ extending $p \notin S$ such that $F_{w}=F_{\infty}$. According to the above choices then $H^{1}\left(F_{\infty}, \mu(\mathbb{C})\right)=H^{1}\left(F_{w}, \mu\left(k_{w}\right)\right) \stackrel{\sim}{\rightarrow} H^{1}\left(\mathbb{F}_{q} / \mathbb{F}_{p}, \mu\left(\mathbb{F}_{q}\right)\right)$. If now $U_{p} \subset G\left(\mathbb{Z}_{p}\right)$ is the full congruence subgroup $\bmod p$ then the commutative diagrams show that $\Gamma \cap U_{p}=\Gamma^{\prime}$ has the desired property $\partial_{\infty}\left(\Gamma^{\prime}\right)=1$.

Remark. Let us assume that $\Gamma$ has the congruence subgroup property, i.e., for every subgroup $\Gamma^{\prime} \subset \Gamma$ of finite index there exists a congruence subgroup $\Gamma^{\prime \prime}$ of $\Gamma$ such that $\Gamma^{\prime \prime} \subset \Gamma^{\prime}$. Then Proposition 2.2 holds trivially. However, if $H \subset G$ is a $\mathbb{Q}$-rational subgroup then $\Gamma \cap H(\mathbb{Q})$ does not necessarily have the congruence subgroup property. This means that we cannot apply this sort of argument simultaneously to $G$ and finitely many subgroups $H_{i}$. This case is dealt with in the following

Proposition 2.3. Let $G$ be an algebraic group defined over $\mathbb{Q}$, and let $\Theta$ be a finite group of $\mathbb{Q}$-rational automorphisms of $G$. Assume that $\Gamma \subset G(\mathbb{Q})$ is a $\Theta$ stable arithmetic subgroup of $G$. Then there exists a normal $\Theta$-stable torsionfree arithmetic subgroup $\Gamma_{1}$ of $\Gamma$ such that for all cocycles $\gamma$ for $H^{1}\left(\Theta, \Gamma_{1}\right)$ one has $\Gamma_{1}(\gamma) \subset G(\gamma)(\mathbb{R})^{0}$. 
Proof. The first nonabelian cohomology set $H^{1}(\Theta, \Gamma)$ is finite (cf. $[3,3.8]$ ). We choose cocycles $\gamma^{1}, \ldots, \gamma^{t}$ representing the different classes in $H^{1}(\Theta, \Gamma)$. Using the result Proposition 2.2 we can find a congruence subgroup $\Gamma_{0} \subset \Gamma$ such that

$$
\Gamma_{0} \cap G\left(\gamma^{i}\right)(\mathbb{Q}) \subset G\left(\gamma^{i}\right)(\mathbb{R})^{0}, \quad i=1, \ldots, t,
$$

holds; we may assume that $\Gamma_{0}$ is torsion free [23]. Then we put $\Gamma_{1}=\bigcap_{s \in \Theta^{S}} s(\Gamma)$ and, in case $\Gamma_{1}$ is not normal in $\Gamma$, we replace $\Gamma_{1}$ by $\bigcap \eta \Gamma_{1} \eta^{-1}, \eta \in \Gamma / \Gamma_{1}$. Now our claim follows from the following observation: If $\gamma$ and $\delta$ are two cocycles representing different classes in $H^{1}\left(\Theta, \Gamma_{1}\right)$ but becoming equivalent in $H^{1}(\Theta, \Gamma)$ then there is an element $\alpha$ in $\Gamma$, such that $\gamma_{s}=\alpha^{-1} \delta_{s}{ }^{s} \alpha$ for all $s \in \Theta$. Then one has isomorphisms $\operatorname{int}(\alpha): \Gamma_{1}(\gamma) \stackrel{\sim}{\rightarrow} \Gamma_{1}(\delta)$ and $\operatorname{int}(\alpha)$ : $G(\gamma)(\mathbb{R})^{0} \stackrel{\sim}{\rightarrow} G(\delta)(\mathbb{R})^{0}$.

As in $\S 1$ we now consider the group $\Theta$ generated by two rational $\mathbb{Q}$-automorphisms $\sigma, \tau$ of $G(\mathbb{Q})$ of finite order which commute with each other. Given a cocycle $\gamma=\left(\gamma_{s}\right), s \in \boldsymbol{\Theta}$, there are the two cocycles $\gamma_{\mu}$ for $H^{1}(\mu, \Gamma), \mu=\sigma, \tau$, obtained from $\gamma$ by restriction.

Since the $\gamma$-twisted $\Theta$-action on $X$ is determined by the $\gamma_{\sigma}$-twisted $\sigma$-action together with the $\gamma_{\tau}$-twisted $\tau$-action, we have for the corresponding sets of fixed points $X(\gamma)=X\left(\gamma_{\sigma}\right) \cap X\left(\gamma_{\tau}\right)$. Then we obtain the following

Corollary 2.4. Let $\Gamma \subset G(\mathbb{Q})$ be a $\Theta$-stable arithmetic subgroup of $G$. Then there exists a normal $\Theta$-stable torsionfree arithmetic subgroup $\Gamma_{1}$ of $\Gamma$ such that for all cocycles $\gamma_{s}$ for $H^{1}\left(\Theta, \Gamma_{1}\right)$ the corresponding groups $\Gamma_{1}(\gamma)$ resp. $\Gamma_{1}\left(\gamma_{\sigma}\right)$ resp. $\Gamma_{1}\left(\gamma_{\tau}\right)$ act orientation preserving on $X(\gamma)$ resp. $X\left(\gamma_{\sigma}\right)$ resp. $X\left(\gamma_{\tau}\right)$.

\section{INTERSECTION NUMBERS}

In this section we recall the definition of the intersection number of two closed immersed oriented submanifolds in an oriented manifold and prove a formula describing the local contributions to the intersection number of special cycles in terms of certain Euler numbers.

3.1. Let $N$ be a closed immersed oriented submanifold of an oriented manifold $V$. We choose a tubular neighborhod $U$ of $N$ in $V$. If we have $v=\operatorname{dim} V$ (resp. $n=\operatorname{dim} N$ ) then there is an isomorphism

$$
H^{v-n}(U, U-N ; \mathbb{Z}) \stackrel{\sim}{\rightarrow} H^{v-n}(V, V-N ; \mathbb{Z}) .
$$

Since the normal bundle of $N$ in $V$ is oriented in a natural way, there is a distinguished isomorphism

$$
j_{x}: H^{v-n}\left(U_{x},(U-N)_{x} ; \mathbb{Z}\right) \stackrel{\sim}{\rightarrow} \mathbb{Z}
$$


where $U_{x}$ denotes the fibre of the normal bundle in $U$ over $x \in N$. By Theorem 10.4 of [22] there is a unique class $w_{N}$ in $H^{v-n}(U, U-N ; \mathbb{Z})$ such that $w_{N}$ is mapped under the composite of maps

$$
H^{v-n}(U, U-N ; \mathbb{Z}) \rightarrow H^{v-n}\left(U_{x},(U-N)_{x} ; \mathbb{Z}\right) \stackrel{j_{x}}{\rightarrow} \mathbb{Z}
$$

to the element 1 in $\mathbb{Z}$.

3.2. Let $M, N$ be two closed immersed oriented submanifolds of an oriented manifold $V$ of $\operatorname{dim} V=v$. We assume that the intersection $M \cap N$ is compact and that $\operatorname{dim} M+\operatorname{dim} N=m+n=v$ holds. Then the intersection number of $M$ and $N$ is defined by

$$
[M][N]=\left(w_{M} \cup w_{N}\right)[V]
$$

where the right-hand side denotes the evaluation of the cup product $w_{M} \cup w_{N}$ in $H_{c}^{v}(V ; \mathbb{Z})$ on the fundamental class $[\mathrm{V}]$ of $V$.

Now we assume that the intersection $M \cap N$ consists of one connected (compact) manifold $F$, let us say, of dimension $f \geq 1$. Moreover, we assume that $M$ and $N$ intersect perfectly in the sense of 1.3 . Let $T M_{\mid F}+T N_{\mid F}$ be the bundle over $F$ whose fibre over a point $x \in F$ consists of the span of the fibres $T_{x} M$ and $T_{x} N$ in the fibre $T_{x} V$ of the tangent bundle of $V$. Then there are exact sequences of bundles

$$
0 \rightarrow T F \rightarrow T M_{\mid F} \oplus T N_{\mid F} \rightarrow T M_{\mid F}+T N_{\mid F} \rightarrow 0
$$

and

$$
0 \rightarrow T M_{\mid F}+T N_{\mid F} \rightarrow T V_{\mid F} \rightarrow \eta \rightarrow 0 .
$$

Since $M$ and $N$ intersect perfectly, the sequence (3) defines an $f$-dimensional vector bundle $\eta$ over $F$ given as the quotient of the tangent bundle of $V$ by the sum of the tangent bundles of $M$ and $N$ restricted to $F$. The bundle $\eta$ will be called the excess bundle of the intersection $F$ (for this notion see, e.g., [7]). Note that the excess bundle is zero if the intersection is transversal.

Recall that the manifolds $V, M$, and $N$ are oriented. Suppose that $F$ is orientable, and fix an orientation on $F$. Then the excess bundle is an oriented vector bundle in a natural way. Let $e(\eta)$ be the corresponding Euler class of $\eta$ in $H^{f}(F ; \mathbb{Z})$.

If $[F] \in H_{f}(F, \mathbb{Z})$ denotes the fundamental class of $F$ then evaluation of $e(\eta)$ on $[F]$ gives a well-defined integer

$$
e(\eta)[F]
$$

it is called the Euler number of $\eta$. Note that $e(\eta)[F]$ does not depend on the choice of the orientation of $F$. To have a smooth notation we introduce for 
$F=\{x\}$ the notation $e(\eta)[F]=1$ if $T_{x} M+T_{x} N$ equals $T_{x} V$ as an oriented vectorspace and $e(\eta)[F]=-1$ otherwise.

For the lack of a suitable reference we sketch the proof of the following

Proposition 3.3. Let $M, N$ be two closed immersed oriented submanifolds of an oriented manifold $V$. Suppose that $\operatorname{dim} M+\operatorname{dim} N=\operatorname{dim} V$, that $M$ and $N$ intersect perfectly, and that the intersection $M \cap N$ consists of one connected compact orientable manifold $F$ with excess bundle $\eta$. Then one has

$$
[M][N]=e(\eta)[F] .
$$

Proof. (Sketch) We may replace $V$ (resp. $M, N$ ) by the total space of the normal bundle $\nu_{V}(F)$ (resp. $\nu_{M}(F), \nu_{N}(F)$ ) of $F$ in $V$ (resp. $M, N$ ). We have

$$
\nu_{V}(F)=\nu_{M}(F) \oplus \nu_{N}(F) \oplus \eta
$$

as a direct sum. If $p_{M}$ denotes the projection to the last two summands then $p_{M}^{*}\left(\nu_{N}(F) \oplus \eta\right)$ is the normal bundle of $M$ in $V$. Let $u_{M}$ and $u_{N}$ denote the fundamental cohomology class of $\nu_{M}(F)$ and $\nu_{N}(F)$ respectively, and let $u_{F}$ be the one of $\eta$. Then $p_{M}^{*}\left(u_{N} \cup u_{F}\right)$ is the dual class of the fundamental class of $M$ in $V$, and $p_{N}^{*}\left(u_{M} \cup u_{F}\right)$ is the dual class of the fundamental class of $N$ in $V$ where $p_{N}$ is defined in an analogous way as $p_{M}$.

We have to evaluate the cup product of these two classes on the fundamental class $[V]$. As above we replace $V$ by the total space of the normal bundle $\nu_{V}(F)$ of $F$ in $V$. First we evaluate over the fibres of this bundle and then over the base space $F$. The contributions along the suitable fibre with respect to $u_{M}, u_{N}$, and $u_{F}$ are 1 by definition of these classes. Thus we obtain

$$
[M][N]=\left(s^{*} u_{F}\right)[F]
$$

where $s$ denotes the zero section of the bundle $\eta$. By definition of the Euler class, the right-hand side is equal to $e(\eta)[F]$.

3.4. Let $G$ be a connected reductive algebraic group defined over $\mathbb{Q}$, and let $\boldsymbol{\Theta}=\langle\sigma, \tau\rangle$ be the group generated by two rational $\mathbb{Q}$-automorphisms $\sigma, \tau$ of $G(\mathbb{Q})$ of finite order which commute with each other. We consider a $\Theta$ stable torsionfree arithmetic subgroup $\Gamma$ of $G(\mathbb{Q})$ chosen in such way that the special cycles $C(\sigma), C(\tau)$ and all connected components $F(\gamma), \gamma \in H^{1}(\Theta, \Gamma)$, of their intersection are orientable (cf. Corollary 2.4). We fix orientations on $X / \Gamma, C(\sigma)$, and $C(\tau)$, and we suppose that the intersection $C(\sigma) \cap C(\tau)$ is compact and that $\operatorname{dim} C(\sigma)+\operatorname{dim} C(\tau)=\operatorname{dim} X / \Gamma$ holds. Then the following proposition is a direct consequence of Propositions 1.2 and 3.3.

Proposition 3.5. Under the assumptions of 3.4 and with the notation of 3.2 the intersection number of the two special cycles $C(\sigma)$ and $C(\tau)$ is given by

$$
[C(\sigma)][C(\tau)]=\sum_{\gamma} e(\eta(\gamma))[F(\gamma)]
$$


where the sum ranges over the elements in the kernel of the map

$$
\operatorname{res}_{\sigma} \times \operatorname{res}_{\tau}: H^{1}(\sigma, \tau, \Gamma) \rightarrow H^{1}(\sigma, \Gamma) \times H^{1}(\tau, \Gamma),
$$

and $\eta(\gamma)$ denotes the excess bundle of the intersection component $F(\gamma)$.

Remarks. (i) The assumption that $C(\sigma, \Gamma) \cap C(\tau, \Gamma)$ is compact is fulfilled if and only if for all $\gamma \in \operatorname{ker}\left(\operatorname{res}_{\sigma} \times \operatorname{res}_{\tau}\right)$ the group $G(\gamma)$ is $\mathbb{Q}$-anisotropic. This follows from the compactness criterion (cf. $[1, \S 8]$ ).

(ii) We would like to add at this point that in the unpublished paper [20] (which we got to know in the fall 1990 at the IAS when the major part of our work was already done) Millson observed in a remark [20, p. 44] after dealing with the case $G_{2}$ that the Euler number of the excess bundle might play a role in the study of degenerate intersections. For this connection he refers to the clean intersection formula of Quillen [25, §3]. A similar phenomenon as in the case $G_{2}$ was observed in [18, Proposition 4.9] in dealing with $\mathrm{SL}_{3}$.

\section{The Euler number of THE EXCess Bundle}

In this section we investigate the contribution $e(\eta(\gamma))[F(\gamma)]$ to the intersection number $[C(\sigma)][C(\tau)]$ of two special cycles. In Proposition 4.2 we show that the number $e(\eta(\gamma))[F(\gamma)]$ is proportional to an invariant volume of $F(\gamma)$ and determine the proportionality factor. The main result is Theorem 4.11 which establishes the positivity of the intersection number under quite general assumptions.

In this section $G$ is a connected reductive algebraic group defined over $\mathbb{Q}$.

4.1. As in 3.4 we consider a $\langle\sigma, \tau\rangle$-stable torsionfree arithmetic subgroup $\Gamma$ of $G(\mathbb{Q})$ such that the special cycles $C(\sigma)$ and $C(\tau)$ and the connected components $F(\gamma), \gamma \in \operatorname{ker}\left(\operatorname{res}_{\sigma} \times\right.$ res $\left._{\tau}\right)$, are orientable. We choose orientations on $X / \Gamma, C(\sigma), C(\tau)$ and use the notation of Proposition 3.5. We denote a representing cocycle $\left(\gamma_{s}\right), s \in \boldsymbol{\Theta}=\langle\sigma, \tau\rangle$, for an element $\gamma$ in $\operatorname{ker}\left(\operatorname{res}_{\sigma} \times\right.$ res $\left._{\tau}\right)$ by the same letter. We choose a point $x \in X(\gamma)$ and denote by $K_{x}$ the maximal compact subgroup of $G(\mathbb{R})$ corresponding to $x$. Then the $\gamma$-twisted $\Theta$-action is defined on $K_{x}$ and we have

$$
K_{x}(\gamma) \backslash G(\gamma)(\mathbb{R}) \stackrel{\sim}{\rightarrow} X(\gamma) .
$$

The Cartan decomposition of the Lie algebra $\mathfrak{g}$ of $G(\mathbb{R})$ corresponding to the point $x \in X(\gamma)$ is denoted by

$$
\mathfrak{g}=\mathfrak{k}_{x} \oplus \mathfrak{p}_{x} \text {. }
$$

We obtain an exact sequence endowed with an $K_{x}(\gamma)$-action

$$
0 \rightarrow \mathfrak{p}_{x}\left(\gamma_{\sigma}\right)+\mathfrak{p}_{x}\left(\gamma_{\tau}\right) \rightarrow \mathfrak{p}_{x} \rightarrow \eta_{x} \rightarrow 0
$$

where, by definition, $\eta_{x}$ denotes the cokernel of the inclusion. Since we have $K_{x}(\gamma)^{0} \backslash G(\gamma)(\mathbb{R})^{0} \stackrel{\sim}{\rightarrow} K_{x}(\gamma) \backslash G(\gamma)(\mathbb{R})$, the action of the connected component 
$K_{x}(\gamma)^{0}$ of $K_{x}(\gamma)$ on $\eta_{x}$ determines a bundle $\eta$ over $X(\gamma)$ which is the pull back of the excess bundle $\eta(\gamma)$ over $F(\gamma)$ under the map $X(\gamma) \rightarrow X(\gamma) / \Gamma(\gamma)$.

Let $G_{u}(\gamma) / \mathbb{R}$ be the compact real form of $G(\gamma)$. We consider $G_{u}(\gamma)(\mathbb{R})$ as the maximal compact subgroup of $G(\gamma)(\mathbb{C})$ which is determined by the choice of $x$ such that we have an embedding

$$
X(\gamma)=K_{x}(\gamma)^{0} \backslash G(\gamma)(\mathbb{R})^{0} \hookrightarrow K_{x}(\gamma)^{0} \backslash G_{u}(\gamma)(\mathbb{R})^{0}=: X_{u}(\gamma)
$$

of $X(\gamma)$ into the compact dual $X_{u}(\gamma)$. Now the $K_{x}(\gamma)^{0}$-module $\eta_{x}$ determines a bundle on $X_{u}(\gamma)$, to be denoted by $\eta_{u}(\gamma)$. Clearly, orientations of $X(\gamma)$ and of $\eta(\gamma)$ determine orientations on $X_{u}(\gamma)$ and on $\eta_{u}(\gamma)$. We observe that the dimension of the fibre of $\eta_{u}(\gamma)$ is equal to $f(\gamma):=\operatorname{dim} X_{u}(\gamma)$. Therefore, evaluating the Euler class $e\left(\eta_{u}(\gamma)\right)$ of $\eta_{u}(\gamma)$ on the fundamental class $\left[X_{u}(\gamma)\right]$ of $X_{u}(\gamma)$ gives a well-defined number $e\left(\eta_{u}(\gamma)\right)\left[X_{u}(\gamma)\right]$. We observe that this number only depends on the class represented by $\gamma$.

Let $\omega$ be an invariant positive measure on $G(\gamma)(\mathbb{R})$. If $d k$ denotes the invariant measure on $K_{x}(\gamma)$ having mass 1 then $\omega$ and $d k$ determine a measure $\mu$ on $X(\gamma)$. We may view $\mu$ as a $G(\gamma)(\mathbb{R})$-invariant differential form on $X(\gamma)$. Using the fact that the tangent spaces in $x$ at $X(\gamma)$ and $X_{u}(\gamma)$ coincide we extend $\mu_{x}$ to a $G_{u}(\gamma)(\mathbb{R})$-invariant measure on $X_{u}(\gamma)$. By means of the measure $d k$ on $K_{x}(\gamma)^{0}$ and $K_{x}(\gamma)^{0} \backslash G_{u}(\gamma)(\mathbb{R})^{0}=X_{u}(\gamma)$ we obtain a well-defined measure on $G_{u}(\gamma)(\mathbb{R})$. This measure is called the measure corresponding to $\omega$ and will be denoted by $\omega_{u}$.

In this set-up we have the following result.

Proposition 4.2. Under the assumptions of 4.1 and with the notation explained there one has the formula

$$
e(\eta(\gamma))[F(\gamma)]=(-1)^{f(\gamma) / 2} \frac{e\left(\eta_{u}(\gamma)\right)\left[X_{u}(\gamma)\right]}{\operatorname{vol}_{\omega_{u}}\left(G_{u}(\gamma)(\mathbb{R})^{0}\right)} \int_{G(\gamma)(\mathbb{R})^{0} / \Gamma(\gamma)} \omega,
$$

where $f(\gamma)=\operatorname{dim} F(\gamma)$. Moreover, if $G(\gamma)(\mathbb{R})^{0}$ does not contain a compact Cartan subgroup or if $f(\gamma)$ is odd then $e(\eta(\gamma))[F(\gamma)]=0$.

Proof. We choose a $G(\mathbb{R})$ - and $\Theta$-invariant nondegenerate bilinear form on $\mathfrak{g}$ which induces a $K_{x}$-invariant metric $($,$) on \mathfrak{p}_{x}, x \in X$. We use this metric to split the exact sequence 4.1 (3); i.e., we identify $\eta_{x}, x \in F(\gamma)$ with the orthogonal complement of $\mathfrak{p}_{x}\left(\gamma_{\sigma}\right)+\mathfrak{p}_{x}\left(\gamma_{\tau}\right)$ in $\mathfrak{p}_{x}$. Hence $\eta$ has the structure of a Riemannian vector bundle with corresponding Riemannian connection $\triangle_{\eta}$. The associated curvature tensor $R_{\eta}$ is given by

$$
R_{\eta}(x, y) z=-a d[x, y] z
$$

where $x, y \in \mathfrak{p}_{x}(\gamma)$ and $z \in \eta_{x}$. For these facts, see [8, VII, $\S 7$, VIII, 8.26.2]. We note that $R_{\eta}$ viewed as tensor on $X(\gamma)$ is $G(\gamma)(\mathbb{R})^{0}$-invariant. Hence the Euler class of $e(\eta)$ viewed as a differential form is given by a $G(\gamma)(\mathbb{R})^{0}$-invariant differential form on $X(\gamma)$ of the form $a \omega, a \in \mathbb{R}$. We have to compute $a$. 
For this we use an extension of Hirzebruch's proportionality principle [11] to bundles. We fix $x \in X(\gamma)$ and consider the embedding $X(\gamma) \hookrightarrow X_{u}(\gamma)$ given by the identification $\mathfrak{p}_{x} \stackrel{\sim}{\rightarrow} i \mathfrak{p}_{x}$. The bundle $\eta_{u}$ on $X_{u}(\gamma)$ determined by $i \mathfrak{p}_{x}$ and the $K_{x}(\gamma)^{0}$ action on $i \mathfrak{p}_{x}$ has as above a Riemannian connection $R_{\eta_{u}}$. Using the identification $\mathfrak{p}_{x} \stackrel{\sim}{\rightarrow} i \mathfrak{p}_{x}$ one has

$$
R_{\left.\eta\right|_{x}}=-R_{\left.\eta_{u}\right|_{x}}
$$

As above we have for the Euler class $e\left(\eta_{u}\right)=b \omega_{u}$ where $b \in \mathbb{R}$. The Euler class of an odd-dimensional bundle is zero [14, $\S 16,7.7]$. So let us assume that $f(\gamma)$ is even. From $(5)$ we deduce that $a=(-1)^{f(\gamma) / 2} \cdot b$. But, of course,

$$
e\left(\eta_{u}\right)\left[X_{u}(\gamma)\right]=\int_{G_{u}(\gamma)(\mathbb{R})^{0}} b \omega_{u}
$$

and the formula given in Proposition 4.2 follows. Now $e\left(\eta_{u}(\gamma)\right)\left[X_{u}(\gamma)\right]=0$ if $G(\gamma)(\mathbb{R})^{0}$ has no compact Cartan subgroup [8, IX, 9.15]. Therefore, all claims have been proved.

Remark. (i) For the Euler number $\chi(F(\gamma))$ of $F(\gamma)$ we get in the same way

$$
\chi(F(\gamma))=(-1)^{f(\gamma) / 2} \frac{\chi\left(X_{u}(\gamma)\right)}{\operatorname{vol}_{\omega_{u}}\left(G_{u}(\gamma)(\mathbb{R})^{0}\right)} \int_{G(\gamma)(\mathbb{R})^{0} / \Gamma(\gamma)} \omega
$$

(ii) The number $\left|e\left(\eta_{u}(\gamma)\right)\left[X_{u}(\gamma)\right]\right|$ only depends on the image of $\gamma$ in $H^{1}(\sigma, \tau, G(\mathbb{R}))$.

4.3. Let $\gamma, \xi \in \operatorname{ker}\left(\right.$ res $_{\sigma} \times$ res $\left._{\tau}\right)$ be classes which map to the same element in $H^{1}(\sigma, \tau, G(\mathbb{R}))$. If $\gamma_{s}$ resp. $\xi_{s}$ are cocycles which represent $\gamma$ resp. $\xi$ then this means that there is a $g \in G(\mathbb{R})$ such that $\gamma_{s}=g^{-1} \xi_{s}{ }^{s} g, s \in\langle\sigma, \tau\rangle$. It follows that right translation by $g$ induces an isomorphism

$$
R(g): X(\xi) \stackrel{\sim}{\rightarrow} X(\gamma)
$$

and also an isomorphism

$$
r(g): X\left(\xi_{\sigma}\right) \times X\left(\xi_{\tau}\right) \times X \stackrel{\sim}{\rightarrow} X\left(\gamma_{\sigma}\right) \times X\left(\gamma_{\tau}\right) \times X
$$

We recall that $\Gamma$ has been chosen such that $\Gamma(\sigma), \Gamma(\tau)$, and $\Gamma$ act orientation preserving on their symmetric space $X(\sigma), X(\tau)$, and $X$ and that we have chosen orientations on $X(\sigma), X(\tau)$, and $X$; (see Corollary 2.4 and 3.4, 4.1). We have $\gamma_{\sigma}=a^{-1 \sigma} a, \gamma_{\tau}=b^{-1 \tau} b$ with $a, b \in \Gamma$ and $a$ resp. $b$ are unique modulo $\Gamma(\sigma)$ resp. $\Gamma(\tau)$. We get that right translation with $a$ resp. $b$ induces isomorphism

$$
X(\sigma) \stackrel{\sim}{\rightarrow} X\left(\gamma_{\sigma}\right) \quad \text { resp. } X(\tau) \stackrel{\sim}{\rightarrow} X\left(\gamma_{\tau}\right) .
$$

These maps induce an orientation on $X\left(\gamma_{\sigma}\right)$ resp. $X\left(\gamma_{\tau}\right)$ such that the maps become orientation preserving. Since $\Gamma(\sigma)$ resp. $\Gamma(\tau)$ act orientation preserving the induced orientations do not depend on the choice of $a$ resp. $b$. 
Given cocycles $\gamma_{s}$ and $\xi_{s}$ the equation $\gamma_{s}=g^{-1} \xi_{s}{ }^{s} g$ determines $g$ up to a factor $a \in G\left(\xi_{s}\right)(\mathbb{R})$.

We now define $s(\gamma, \xi)$ as follows: If there is an a $\in G\left(\xi_{s}\right)(\mathbb{R})$ such that the right translation $r(a)$ acts orientation reversing on $X\left(\xi_{\sigma}\right) \times X\left(\xi_{\tau}\right) \times X$, we put $s(\gamma, \xi)=0$. If all $a \in G\left(\xi_{s}\right)(\mathbb{R})$ act orientation preserving, we put $s(\gamma, \xi)=1$ if $r(g)$ acts orientation preserving and $s(\gamma, \xi)=-1$ otherwise. We note that $s(\gamma, \xi)$ only depends on the classes $\gamma, \xi \in \operatorname{ker}\left(\operatorname{res}_{\sigma} \times \operatorname{res}_{\tau}\right)$. This follows from the fact that $\Gamma(\sigma), \Gamma(\tau)$, and $\Gamma$ act orientation preserving on $X(\sigma), X(\tau)$, and $X$.

Proposition 4.4. If the classes $\gamma, \xi \in \operatorname{ker}\left(\operatorname{res}_{\sigma} \times\right.$ res $\left._{\tau}\right)$ map to the same class in $H^{1}(\sigma, \tau, G(\mathbb{R}))$ then

$$
e\left(\eta_{u}(\gamma)\right)\left[X_{u}(\gamma)\right]=s(\gamma, \xi) e\left(\eta_{u}(\xi)\right)\left[X_{u}(\xi)\right] .
$$

Proof. We choose cocycles $\gamma=\left\{\gamma_{s}\right\}$ resp. $\xi=\left\{\xi_{s}\right\}$ representing $\gamma$ resp. $\xi$ and write $\gamma_{s}=g^{-1} \xi_{s}{ }^{s} g, s \in \Theta, g \in G(\mathbb{R})$, as in 4.3. We fix $x \in X(\xi)$. Then $x g \in X(\gamma)$. We have Cartan decompositions $\mathfrak{g}=\mathfrak{k}_{x} \oplus \mathfrak{p}_{x}$ resp. $\mathfrak{g}=\mathfrak{k}_{x g} \oplus \mathfrak{p}_{x g}$ and right translation with $g$ induces an isomorphism

$$
\operatorname{Ad}\left(g^{-1}\right): \mathfrak{p}_{x} \rightarrow \mathfrak{p}_{x g}
$$

Moreover, $\operatorname{Ad}\left(g^{-1}\right)$ induces an isomorphism

$$
f(g): \mathfrak{p}_{x}\left(\xi_{\sigma}\right) \oplus \mathfrak{p}_{x}\left(\xi_{\tau}\right) \oplus \mathfrak{p}_{x} \stackrel{\sim}{\rightarrow} \mathfrak{p}_{x g}\left(\gamma_{\sigma}\right) \oplus \mathfrak{p}_{x g}\left(\gamma_{\tau}\right) \oplus \mathfrak{p}_{x g}
$$

We write $\eta_{\gamma}$ resp. $\eta_{\xi}$ for the pullback of the excess-bundles $\eta(\gamma)$ resp. $\eta(\xi)$ to $X(\gamma)$ resp. $X(\xi)$. Let $\eta_{x}(\xi)$ be the fibre of $\eta_{\xi}$ over $x \in X(\xi)$. Then $\eta_{\xi}$ is determined by the $K_{x}(\xi)^{0}$ action on $\eta_{x}(\xi)$. In the same way $\eta_{\gamma}$ is determined by the $K_{x g}(\gamma)^{0}$ action on $\eta_{x g}(\gamma)$. The definition of these fibres in 4.1 (3) shows that $\operatorname{Ad}\left(g^{-1}\right)$ induces on isomorphism

$$
F(g): \eta_{\xi} \rightarrow \eta_{\gamma}
$$

where $F(g)$ induces the isomorphism $X(\xi) \rightarrow X(\gamma)$ of the base spaces given by right translation with $g$.

Using the exponential map we see that (1) induces the map $r(g): X\left(\xi_{\sigma}\right) \times$ $X\left(\xi_{\tau}\right) \times X \rightarrow X\left(\gamma_{\sigma}\right) \times X\left(\gamma_{\tau}\right) \times X$; see $4.3(1)$. Hence $r(g)$ is orientation preserving if and only if $f(g)$ is orientation preserving. The tangent space at $x$ to the total space of $\eta_{\xi}$ is $\mathfrak{p}_{x}(\xi) \oplus \eta_{x}(\xi)$. The orientation of this space is uniquely determined by the orientation of $\mathfrak{p}_{x}\left(\xi_{\sigma}\right) \oplus \mathfrak{p}_{x}\left(\xi_{\tau}\right)$ and of $\mathfrak{p}_{x}$. Hence $F(g)$ is orientation preserving if and only if $f(g)$ is orientation preserving. Now $F(g)$ is orientation preserving if the map induced by $F(g)$ on the corresponding bundles $\eta_{u}(\xi)$ and $\eta_{u}(\gamma)$ over $X_{u}(\xi)$ is orientation preserving. Therefore, our claim holds provided $s(\gamma, \xi) \neq 0$. 
Suppose that $s(\gamma, \xi)=0$. Then there is an $a \in G(\xi)(\mathbb{R})$ and hence an $a \in K_{x}(\xi)$ acting orientation reversing on the total space of $\eta_{u}(\xi)$. Denote by $\eta_{u}\left(\xi_{-}\right)$the total space of $\eta_{u}(\xi)$ with the opposite orientation of the fibres. Then $a$ induces an orientation-preserving map $\eta_{u}(\xi) \stackrel{\sim}{\rightarrow} \eta_{u}\left(\xi_{-}\right)$. We get $e\left(\eta_{u}(\xi)\right)\left[X_{u}(\xi)\right]=e\left(\eta_{u}\left(\xi_{-}\right)\right)\left[X_{u}(\xi)\right]$. On the other hand, $e\left(\eta_{u}\left(\xi_{-}\right)\right)\left[X_{u}(\xi)\right]=$ $-e\left(\eta_{u}(\xi)\right)\left[X_{u}(\xi)\right]$. Hence this number is zero. Since the same argument applies if $\xi$ is replaced $\gamma$, our claim holds.

We will show that $s(\gamma, \xi)=1$ holds in many cases. For this we use the following observation.

Lemma 4.5. Assume that $G(\mathbb{R})$ resp. $G(\sigma)(\mathbb{R})$ resp. $G(\tau)(\mathbb{R})$ act orientation preserving on $X$ resp. $X(\sigma)$ resp. $X(\tau)$. If $\xi, \gamma$ are as in 4.3 , then $s(\gamma, \xi)=1$. Proof. Let $g_{s}, s \in\langle\sigma, \tau\rangle$ be a cocycle for

$$
\operatorname{ker}\left(H^{1}(\sigma, \tau, G(\mathbb{R})) \rightarrow H^{1}(\sigma, G(\mathbb{R})) \times H^{1}(\tau, G(\mathbb{R}))\right),
$$

i.e., $g_{\sigma}=a^{-1 \sigma} a$ and $g_{\tau}=b^{-1 \tau} b, a, b \in G(\mathbb{R})$. Then $X\left(g_{\sigma}\right)=X(\sigma) a$ and $X\left(g_{\tau}\right)=X(\tau) b$ carry a well-defined orientation such that right translation with $a$ resp. $b$ gives orientation-preserving maps

$$
X(\sigma) \stackrel{\sim}{\rightarrow} X\left(g_{\sigma}\right), \quad X(\tau) \stackrel{\sim}{\rightarrow} X\left(g_{\tau}\right) .
$$

It follows that the map $r(g)$ of 4.3. (1) always is orientation preserving.

Proposition 4.6. Let $K$ be a connected simply connected compact Lie group. If $\sigma$ is an automorphism of finite order of $K$, then $K(\sigma)$ is connected.

Proof. There exists a linear algebraic group $G_{0}$ over $\mathbb{R}$ such that $K=G_{0}(\mathbb{R})$ (see [6, Chapter VI, §9]) and $\sigma$ induces an $\mathbb{R}$-rational automorphism $\sigma$ of $G_{0}$. Then $K$ is a compact real form of $G_{0}(\mathbb{C})$ and $K$ is a maximal compact subgroup of $G_{0}(\mathbb{C})$. The corresponding Cartan decomposition shows that $G_{0}(\mathbb{C})$ is connected and simply connected as locally compact topological group. Hence $G_{0}$ is (Zariski)-connected and simply connected as an algebraic group. According to [32, Theorem 8.1] $G_{0}(\sigma)$ is a (Zariski)-connected reductive group defined over $\mathbb{R}$. Since $G_{0}(\sigma)(\mathbb{R})=K(\sigma)$ is compact, the group $G_{0}(\sigma)(\mathbb{R})$ is connected, cf. $[1,11.22]$.

Remark. If $\sigma$ is an involution the assertion in Proposition 4.6 is proved in [10, Chapter VII, 7.2] by an entirely different method.

Corollary 4.7. Let $G$ be a connected semisimple and simply connected algebraic group defined over $\mathbb{R}$. Assume that $\sigma$ is an automorphism of finite order of $G$ which is defined over $\mathbb{R}$. If $G(\mathbb{R})$ is simply connected as a topological group, then $G(\sigma)(\mathbb{R})$ is connected.

Proof. We choose a maximal $\sigma$-stable compact subgroup $K$ of $G(\mathbb{R})$. The corresponding Cartan decomposition shows that $K$ is a deformation retract of $G(\mathbb{R})$ and that $K(\sigma)$ is a deformation retract of $G(\sigma)(\mathbb{R})$. By Corollary 4.7 in 
[5] the group $G(\mathbb{R})$ is connected. Since $G(\mathbb{R})$ is simply connected by assumption, we see that $K$ is connected and simply connected. By Proposition 4.6 we have that $K(\sigma)$ is connected. Hence $G(\sigma)(\mathbb{R})$ is connected.

4.8. Let $G / \mathbb{R}$ be a connected simply connected semisimple algebraic group defined over $\mathbb{R}$, and let $\sigma: G \rightarrow G$ be an $\mathbb{R}$-algebraic automorphism of finite order. We now give conditions on $G$ and $\sigma$ such that $G(\sigma)(\mathbb{R})$ acts orientation preserving on $X(\sigma)$.

(i) Cyclic base change. If $G_{0} / k$ is a semisimple connected and simply connected group defined over a number field $k$ and if $\ell / k$ is a cyclic Galois extension with Galois group $\operatorname{gal}(\ell / k)=(\sigma)$ generated by $\sigma$ then $\sigma$ acts on $G=\operatorname{Res}_{\ell \mid \mathbb{Q}}\left(G_{0} \times{ }_{k} \ell\right)$ and $G(\sigma)(\mathbb{R})=G_{0}(k \otimes \mathbb{R})$ is connected by [4, 4.7].

(ii) Hermitian symmetric spaces. It is known that $X=K \backslash G(\mathbb{R})$ is hermitian symmetric if and only if $K$ has a (central) direct factor $S^{1}=\operatorname{SO}(2)(\mathbb{R})$. Then $\sigma$ acts on $S^{1}$ and $G(\sigma)(\mathbb{R})$ acts analytically on $X(\sigma)$ if and only if $\sigma_{\mid S^{1}}=$ Id. In particular, if $\sigma_{\mid S^{1}}=$ Id then $G(\sigma)(\mathbb{R})$ acts orientation preserving on $X(\sigma)$.

(iii) General case. We write $G(\mathbb{R})=G_{\mathrm{c}} \times G_{\mathrm{h}} \times G_{\mathrm{r}}$ where $G_{\mathrm{c}}$ is the product of the compact simple factors of $G(\mathbb{R})$ and $G_{\mathrm{h}}$ is the product of the factors which give rise to hermitian symmetric spaces. The rest of the factors makes up $G_{\mathrm{r}}$. Then $\sigma=\sigma_{\mathrm{c}} \times \sigma_{\mathrm{h}} \times \sigma_{\mathrm{r}}$ and $X=X_{\mathrm{h}} \times X_{\mathrm{r}}$ in obvious notation. The group $G_{\mathrm{r}}\left(\sigma_{\mathrm{r}}\right)$ acts orientation preserving on $X_{\mathrm{r}}\left(\sigma_{\mathrm{r}}\right)$ if the simple factors of $G_{\mathrm{r}}$ are not of the following type: $\mathrm{SL}_{n}(\mathbb{R}), n \geq 3$; $\mathrm{SO}_{n}(q)(\mathbb{R})$ where $q$ is a quadratic form of index $\geq 3$; split $G_{2}, F_{4}, E_{6}, E_{7}, E_{8} ; E_{6,2}, E_{7,-5}, E_{8,-24}$ where the second index is the Cartan index. If the factors of $G(\mathbb{R})$ are not of this type the list in [33] shows that $G(\mathbb{R})$ is simply connected. Hence Corollary 4.7 applies and gives our claim.

Example. Let $G(\mathbb{R})=\mathrm{SL}_{n}(\mathbb{R})$, and let $\sigma$ be the automorphism given by conjugation with the diagonal matrix $\eta=(-1, \ldots,-1,1, \ldots, 1)$, where $k$ is the number of entries -1 and $1 \leq k \leq n-k<n$. If $n$ is odd then the action of $G(\sigma)(\mathbb{R})=\mathrm{SO}(k, n-k)(\mathbb{R})$ on $X(\sigma)$ does not preserve the orientation.

Next we consider the absolute value of the contribution of $F(\gamma)$ to the intersection number in the general setting of 3.4.

Proposition 4.9. Assume that the special cycles $C(\sigma)$ and $C(\tau)$ intersect transversally in at least one point. Then for the absolute value of $e\left(\eta_{u}(\gamma)\right)\left[X_{u}(\gamma)\right]$ we have the identity

$$
\left|e\left(\eta_{u}(\gamma)\right)\left[X_{u}(\gamma)\right]\right|=\chi\left(X_{u}(\gamma)\right) .
$$

Proof. Let $x \in X$ represent a point where $C(\sigma)$ and $C(\tau)$ intersect transversally. We assume that $G(\gamma)(\mathbb{R})$ contains a compact Cartan subgroup; otherwise, there is nothing to prove since both numbers are zero (see Proposition 4.2 and [13]). According to the remark following Proposition 1.2 we can view the element $\gamma \in \operatorname{ker}\left(\right.$ res $_{\sigma} \times$ res $\left._{\tau}\right)$ as an element in the kernel of the map $H^{1}(\sigma, \Gamma(\tau)) \rightarrow H^{1}(\sigma, \Gamma)$. We have $\Gamma(\tau) \subset G(\tau)(\mathbb{R})^{0}$, and there is an isomorphism 


$$
H^{1}\left(\sigma, G(\tau)(\mathbb{R})^{0}\right) \stackrel{\leftarrow}{\leftarrow} H^{1}\left(\sigma, K(\tau)^{0}\right)
$$

where $K$ is the maximal compact subgroup corresponding to $x$. (The argument given in [26, 1.4] works in this case as well.) If $T \subset K(\tau)^{0}$ is a maximal $\sigma$-stable torus and if $S:=T(\sigma)^{0}$ then the inclusion $S \rightarrow K(\tau)^{0}$ induces a surjection

$$
H^{1}(\sigma, S) \rightarrow H^{1}\left(\sigma, K(\tau)^{0}\right) .
$$

We refer to [32, Theorem 7.5] for the existence of $T$ and to [28] for the proof of this claim. Of course, $\Theta=\langle\sigma, \tau\rangle$ acts on $S$. We find that if $\gamma$ is represented by the cocycle $\left(\gamma_{s}\right), s \in \Theta$, then there are a cocycle $\left\{t_{s}\right\}, s \in \Theta, t_{s} \in S$, and an element $g \in G(\mathbb{R})^{0}$ such that

$$
\gamma_{s}=g^{-1} t_{s}^{s} g, \quad s \in \Theta
$$

Conjugation with $g$ shows that it is sufficient to understand the number $\left|e\left(\eta_{u}(t)\right)\left[X_{u}(t)\right]\right|$ in obvious notation.

Let $C_{K}(S)^{0}$ be the connected centralizer of $S$ in $K$. Then $\Theta$ acts on $C_{K}(S)^{0}$ and we find a $\sigma$-stable maximal torus $T_{\sigma}$ in $C_{K}(S)^{0}$; see [32, Theorem 7.5]. Indeed, $T_{\sigma}$ is a maximal torus in $K$. Similarly, we find a $\tau$-stable maximal torus $T_{\tau}$. By construction we have $S=T_{\sigma}(\sigma)^{0}=T_{\tau}(\tau)^{0}$. Since $t_{s} \in S \subset K, s \in \Theta$, one has $x \in X(t)$. We get an equation of $S$-modules

$$
\mathfrak{p}_{x}\left(t_{\sigma}\right) \oplus \mathfrak{p}_{x}\left(t_{\tau}\right) \oplus \eta_{x}(t)=\mathfrak{p}_{x} \oplus \mathfrak{p}_{x}(t)
$$

Now $t_{\sigma}$ represents a class in the kernel of the map $H^{1}\left(\sigma, T_{\sigma}\right) \rightarrow H^{1}(\sigma, K)$. But then $t_{\sigma}$ bounds in the normalizer $N_{K}\left(T_{\sigma}\right)$ of $T_{\sigma}$ in $K$ (cf. [28]). Hence we have $t_{\sigma}=n^{-1 \sigma} n$ for some $n \in N_{K}\left(T_{\sigma}\right)$, and $n$ determines an element $\bar{n}=: w$ in $\left(N_{K}\left(T_{\sigma}\right) / T_{\sigma}\right)^{\sigma}$. Therefore, $w$ acts on $\left(T_{\sigma}(\sigma)\right)^{0}=S$. We write ${ }^{w} \mathfrak{p}_{x}(\sigma)$ for the $w$-twisted $S$-module $\mathfrak{p}_{x}(\sigma)$; i.e., an element $h \in S$ acts on $Y \in \mathfrak{p}_{x}(\sigma)$ via $h Y=w(h) Y$. Conjugation with $n$ induces an isomorphism of $S$-modules

$$
\mathfrak{p}_{x}\left(t_{\sigma}\right) \stackrel{\sim}{\rightarrow} \mathfrak{p}_{x}(\sigma)
$$

Now $w$ permutes the weights of $\mathfrak{p}_{x}(\sigma)$ with respect to $S$, and hence we obtain an isomorphism of $S$-modules

$$
\mathfrak{p}_{x}\left(t_{\sigma}\right) \stackrel{\sim}{\rightarrow} \mathfrak{p}_{x}(\sigma)
$$

The same type of argument applies to the action of $\tau$. By assumption, $X(\sigma)$ and $X(\tau)$ intersect transversally at $x$; hence, we get an isomorphism of $S$-modules

$$
\mathfrak{p}_{x}\left(t_{\sigma}\right) \oplus \mathfrak{p}_{x}\left(t_{\tau}\right) \stackrel{\sim}{\rightarrow} \mathfrak{p}_{x}(\sigma) \oplus \mathfrak{p}_{x}(\tau) \stackrel{\sim}{\rightarrow} \mathfrak{p}_{x}
$$

From (7) and (4) we get an isomorphism of $S$-modules

$$
\eta_{x}(t) \stackrel{\sim}{\rightarrow} \mathfrak{p}_{x}(t)
$$


The characteristic classes of the bundle $\eta_{u}\left(t_{s}\right)$ over $X_{u}\left(t_{s}\right)$ are determined by the weight structure with respect to $S$ (see $[2, \S 10])$. Therefore, we get

$$
\left|e\left(\eta_{u}(t)\right)\left[X_{u}(t)\right]\right|=\left|\chi\left(X_{u}(t)\right)\right| .
$$

Here we use that the Euler class for the tangent bundle given by $\mathfrak{p}_{x}(t)$ determines the Euler characteristic of $X_{u}(t)$ (cf. $[14, \S 17,7.2]$ ). Observe that we are forced to use absolute values in (9) since we work with orientable bundles instead of oriented bundles. By [13], $\chi\left(X_{u}(t)\right)$ is positive.

Remark 4.10. (i) The element $g$ in (3) of the proof of 4.9 may give rise to an orientation reversing $r(g)$; see 4.3. Therefore, $e\left(\eta_{u}(t)\right)\left[X_{u}(t)\right]$ is well defined only up to sign. If $r(g)$ acts orientation preserving the proof of Proposition 4.9 gives $e\left(\eta_{u}(\gamma)\right)\left[X_{u}(\gamma)\right]=\chi\left(X_{u}(\gamma)\right)$.

(ii) Let us denote by $\phi(\zeta)$ a characteristic class of an even-dimensional oriented real vector bundle $\zeta$ over a manifold $Y$ in the ring $H^{*}(Y, \mathbb{C})$, and assume that $\phi(\zeta)$ is invertible in $H^{*}(Y, \mathbb{C})$ and that $\phi()$ is multiplicative with respect to the direct sum of bundles; see [14, Chapter 16, §7]. Denote the tangent bundle of $Y$ by $T Y$. We assume that $r(g), g$ as in (1), acts orientation preserving. Then we have shown in the proof of Proposition 4.9 that without any assumptions about transversality one has

$$
\phi\left(\eta_{u}(t)\right)=\frac{\phi\left(T X_{u \mid X_{u}(t)}\right)}{\phi\left(T X_{u}(\sigma)_{\mid X_{u}(t)}\right) \phi\left(T X_{u}(\tau)_{\mid X_{u}(t)}\right)} \phi\left(T X_{u}(t)\right)
$$

Now we can formulate our main result. For the convenience of the reader we recall the general setting: Let $G$ be a connected reductive algebraic group defined over $\mathbb{Q}$, and let $\sigma, \tau$ be two rational automorphisms of $G(\mathbb{Q})$ of finite order which commute with each other. Let $\Gamma$ be a $\langle\sigma, \tau\rangle$-stable torsionfree arithmetic subgroup of $G(\mathbb{Q})$ chosen in such a way that the special cycles $C(\sigma, \Gamma)$ and $C(\tau, \Gamma)$ and all connected components of their intersection are orientable (cf. $\S 2)$. We suppose that $C(\sigma, \Gamma) \cap C(\tau, \Gamma)$ is compact and that $\operatorname{dim} C(\sigma, \Gamma)+\operatorname{dim} C(\tau, \Gamma)=\operatorname{dim} X / \Gamma$ holds. We say that the condition (Or) is satisfied if $G(\sigma)(\mathbb{R})$ resp. $G(\tau)(\mathbb{R})$ resp. $G$ act orientation preserving on $X(\sigma)$ resp. $X(\tau)$ resp. $X$. In 4.8 conditions on $G$ and automorphisms of finite order are discussed which imply the assertion of (Or).

Theorem 4.11. Let $G, \sigma, \tau$, and $\Gamma$ be as above such that condition (Or) holds. Assume in addition that $G$ is semisimple and that $X(\sigma)$ and $X(\tau)$ intersect in exactly one point with positive intersection number. Then there exists $a\langle\sigma, \tau\rangle-$ stable normal subgroup $\Gamma_{1}$ of finite index in $\Gamma$ such that

$$
\left[C\left(\sigma, \Gamma_{1}\right)\right]\left[C\left(\tau, \Gamma_{1}\right)\right]=\sum \chi(F(\gamma))
$$

where the sum ranges over the elements $\gamma$ in the kernel of the map res $_{\sigma} \times$ $\operatorname{res}_{\tau}: H^{1}\left(\sigma, \tau, \Gamma_{1}\right) \rightarrow H^{1}\left(\sigma, \Gamma_{1}\right) \times H^{1}\left(\tau, \Gamma_{1}\right)$ and where all Euler characteristics $\chi(F(\gamma))$ of the corresponding fixed point components are positive. 
Proof. Our assumption that (Or) holds shows that Remark 4.10(i) applies. Hence we can use Propositions 3.5 and 4.9. For a discussion of the condition (Or) see 4.8. We get

$$
\left[C\left(\sigma, \Gamma_{0}\right)\right]\left[C\left(\tau, \Gamma_{0}\right)\right]=\sum \chi(F(\gamma))
$$

where the sum ranges over the elements $\gamma$ in

$$
\operatorname{ker}\left(H^{1}\left(\sigma, \tau, \Gamma_{0}\right) \rightarrow H^{1}\left(\sigma, \Gamma_{0}\right) \times H^{1}\left(\tau, \Gamma_{0}\right)\right)
$$

The sign of $\chi(F(\gamma))$ is $(-1)^{f(\gamma) / 2}$, by the remark following Proposition 4.2 where $f(\gamma)=\operatorname{dim} F(\gamma)$. Following the arguments in [29, §2], we may replace $\Gamma_{0}$ by a $\langle\sigma, \tau\rangle$-stable subgroup $\Gamma_{1}$ which is normal in $\Gamma$ such that all fixed point components with respect to $\Gamma_{1}$ have the same dimension modulo 4 and such that $\chi(F(\gamma)) \neq 0$. This implies our claim.

4.12. The assumption in Theorem 4.11 that $X(\sigma)$ and $X(\tau)$ intersect in exactly one point with positive intersection number is satisfied if and only if the group $G(\sigma, \tau)(\mathbb{R})$ of fixed points of $\langle\sigma, \tau\rangle$ is compact. Note that the positivity of the intersection number is always easily achieved by rearranging the orientation on $X, X(\sigma)$, and $X(\tau)$. We point out that Millson [20] has constructed an example of $\Gamma, \sigma, \tau$ where $\Gamma \subset \mathrm{SL}_{5}(\mathbb{R})$ such that for all $\langle\sigma, \tau\rangle$-stable arithmetic subgroups sign-changes can occur, i.e., $s(\gamma, \xi)=-1$ in the notation of 4.3 and Proposition 4.4.

\section{REMARKS AND APPLICATIONS}

In this section we discuss some implications of our results for a question raised in the work of Millson and Raghunathan and give an application and explain how our results fit into an adelic approach to the problem.

5.1. Nonvanishing results. The main focus of the work of Millson and Raghunathan $[20,21]$, where in specific cases the intersections of cycles defined by involutions were considered, is the geometric construction of (co)homology classes for arithmetic groups in real Lie groups. They consider an isometry $\psi$ of order 2 of a semisimple noncompact Lie group $G$ and a $\psi$-stable discrete subgroup $\Gamma_{1}$ of $G$ and suppose that the quotient $X / \Gamma_{1}$ is compact. Millson-Raghunathan conjectured (cf. $[19, \mathrm{I}, 4 ; 20])$ that there exists a torsionfree subgroup $\Gamma$ of finite index in $\Gamma_{1}$ such that the special cycle $C(\psi, \Gamma)$ is not a boundary. They proved the conjecture in certain cases of uniform arithmetic subgroups of specific semisimple Lie groups (cf. [21]) by studying the intersection number of $C(\psi, \Gamma)$ with a complementary special cycle $C\left(\theta_{y} \psi, \Gamma\right)$ obtained as described in 1.5 (i). Clearly, the formula for the intersection number of a pair of special cycles obtained in Theorem 4.11 may be used to prove nonvanishing results of this type and to give a partial affirmative answer to this conjecture.

In this respect we discuss as one example the class of hermitian symmetric spaces. 
5.2. Hermitian symmetric spaces. Let us assume that $G, \sigma, \tau, \Gamma$, are as in 3.4. Moreover, let $X$ be hermitian symmetric as in 4.8 (ii) and suppose that $\sigma$ and $\tau$ act analytically on $X$. Then the assumption (Or) in Theorem 4.11 is immediately fulfilled; thus, the cycle $[C(\sigma, \Gamma)]$ is nontrivial in $H_{*}(X / \Gamma, \mathbb{C})$. Recall that this is proved under the assumption that $C(\sigma, \Gamma) \cap C(\tau, \Gamma)$ is compact. If one assumes that $X / \Gamma$ is compact, then it is well known that $C(\sigma, \Gamma)$ and $X / \Gamma$ are in fact projective algebraic varieties. But then the notion of the degree $0 \leq d=d([C(\sigma, \Gamma)])$ is defined and $d>0$ if $\operatorname{dim} C(\sigma, \Gamma) \geq 1$. Clearly this also implies the nonvanishing of the class $[C(\sigma, \Gamma)]$ in $H_{*}(X / \Gamma, \mathbb{C})$. For these algebraic-geometric aspects we refer to [24, 5.35].

5.3. An adelic approach. According to Proposition 4.2 the actual computation of the fixed point contributions to the intersection number in Theorem 4.11 requires volume computations. In view of the now fully established Weil Conjecture on Tamagawa numbers these computations are feasible. They are best done in an adelic setting. The arising formulas then should be simplified in analogy to the stabilisation procedure of the Arthur-Selberg trace formula (see, e.g., [30]). The objects to work with are as follows: Let $K_{f}$ be an open compact $\sigma$-stable subgroup of $G\left(\mathbb{A}_{f}\right)$ where $\mathbb{A}_{f} \subset \mathbb{A}$ denotes the finite adeles in the ring of adeles $\mathbb{A}=\mathbb{A}_{\mathbb{Q}}$. Then it is more natural to work with $C\left(\sigma, K_{f}\right)=G(\sigma)(\mathbb{Q}) \backslash G(\sigma)(\mathbb{A}) /\left(K^{0} K_{f}\right)(\sigma)$ instead of $C(\sigma, \Gamma)$; it is in general nonconnected with an abelian group of components. More generally one considers $\left[C\left(\sigma, K_{f}, \chi\right)\right]=\sum \chi_{i}\left[C_{i}\right]$ where $\chi_{i} \in \mathbb{C}$ and $C_{i}$ are the connected components of $C\left(\sigma, K_{f}\right)$. In this context the dependence on $K_{f}$ of the signs of 4.3 becomes more transparent and the nonvanishing of intersection numbers can often be deduced without the assumption (Or) or the transversality condition.

5.4. A possible generalization. Proposition 3.5 gives an expression for the intersection number $[C(\sigma, \Gamma)][C(\tau, \Gamma)]$ as a sum of fixed point contributions if $C(\sigma, \Gamma) \cap C(\tau, \Gamma)$ is compact. Even without this assumption a similar formula should hold.

\section{REFERENCES}

1. A. Borel, Introduction aux Groupes Arithmetiques, Hermann, Paris, 1969.

2. A. Borel and F. Hirzebruch, Characteristic classes and homogeneous spaces. I, Amer. J. Math. 80 (1958), 458-538.

3. A. Borel and J.-P. Serre, Theoremes de finitude en cohomologie galoisienne, Comment. Math. Helv. 39 (1964), 111-164.

4. A. Borel and J. Tits, Groupes réductifs, Inst. Hautes Études Sci. Publ. Math. 27 (1965), 55-150.

5. _ـ Compléments à l'article: 'Groupes réductifs', Inst. Hautes Études Sci. Publ. Math. 41 (1972), 253-276.

6. C. Chevalley, Theory of Lie groups, Princeton Univ. Press, Princeton, NJ, 1946. 
7. W. Fulton, Intersection Theory, Ergeb. Math. Grenzgeb. (3), vol. 2, Springer, Berlin, Heidelberg, and New York, 1984.

8. W. Greub, S. Halperin, and R. Vanstone, Connections, curvature, and cohomology, 3 vols., Pure Appl. Math., vol. 47, Academic Press, New York and London, 1973.

9. G. Harder, A Gauss-Bonnet formula for discrete arithmetically defined groups, Ann. Sci. École Norm. Sup. (4) 4 (1971), 409-455.

10. S. Helgason, Differential geometry and symmetric spaces, Academic Press, New York and London, 1962

11. F. Hirzebruch, Automorphe Formen und der Satz von Riemann-Roch, Symposium Internacional de Topologia Algebraica (México 1956), La Universidad Nacional Autónoma de México, México, 1958, pp. 129-144.

12. F. Hirzebruch and D. Zagier, Intersection numbers of curves on Hilbert modular surfaces and modular forms of Nebentypus, Invent. Math. 36 (1976), 57-113.

13. H. Hopf and H. Samelson, Ein Satz über die Wirkungsräume geschlossener Lie'scher Gruppen, Comment. Math. Helv. 13 (1940/41), 240-251.

14. D. Husemoller, Fibre Bundles, 2nd ed., Graduate Texts in Math., vol. 20, Springer, New York, Heidelberg, and Berlin, 1975.

15. H. Jacquet and S. Rallis, Symplectic periods, J. Reine Angew. Math. 423 (1992), 175-197.

16. S. Kudla and J. J. Millson, Intersection numbers of cycles on locally symmetric spaces and Fourier coefficients of holomorphic modular forms in several complex variables, Inst. Hautes Études Sci. Publ. Math. 71 (1990), 121-172.

17. S. Lang, Algebraic number theory, Addison-Wesley, Reading, MA, 1970.

18. R. Lee and J. Schwermer, Geometry and arithmetic cycles attached to $S L_{3}(\mathbb{Z})$. I. Topology 25 (1986), 159-174.

19. J. J. Millson, Cycles and harmonic forms on locally symmetric spaces, Canad. Math. Bull. 28 (1985), 3-38.

20. - Geometric construction of homology for arithmetic groups. II, unpublished manuscript, 1981.

21. J. J. Millson and M. S. Raghunathan, Geometric construction of cohomology for arithmetic groups, Geometry and Analysis (Papers dedicated to the memory of Patodi), Indian Academy of Sciences, Bangalore, 1980, pp. 103-123.

22. J. Milnor and J. Stashaff, Characteristic classes, Ann. of Math. Stud., vol. 76, Princeton Univ. Press, Princeton, NJ, 1974.

23. H. Minkowski, Über den arithmetischen Begriff der Äquivalenz und über die endlichen Gruppen linearer ganzzahliger Substitutionen, J. Reine Angew. Math. 100 (1887), 449-458.

24. D. Mumford, Algebraic geometry. I: Complex projective varieties. Grundlehren Math. Wiss., vol. 221, Springer, Berlin, Heidelberg, and New York, 1976.

25. D. Quillen, Elementary proofs of some results of cobordism theory using Steenrod operations, Adv. in Math. 7 (1971), 29-56.

26. J. Rohlfs, Arithmetisch definierte Gruppen mit Galoisoperation, Invent. Math. 48 (1978), 185-205.

27. The Lefschetz number of an involution on the space of classes of positive definite quadratic forms, Comment. Math. Helv. 65 (1981), 272-296.

28. L Lefschetz numbers for automorphisms of finite order on arithmetic groups, in preparation.

29. J. Rohlfs and B. Speh, Automorphic representations and Lefschetz numbers, Ann. Sci. École Norm. Sup. (4) 22 (1989), 473-499.

30. Lefschetz numbers and twisted stabilized orbital integrals, Math. Ann. (to appear).

31. J.-P. Serre, Cohomologie Galoisienne, Lecture Notes in Math. vol. 5, Springer, Berlin, Heidelberg, and New York, 1965. 
32. R. Steinberg, Endomorphisms of linear algebraic groups, Mem. Amer. Math. Soc., vol. 80, Amer. Math. Soc., Providence, RI, 1968.

33. J. Tits, Tabellen zu den einfachen Lie Gruppen und ihren Darstellungen, Lecture Notes in Math., vol. 40, Springer, Berlin, Heidelberg, and New York, 1967.

Katholische Universität Eichstätt, Mathematisch-Geographische FaKUltät, OstenSTRASSE 28, 8078 EICHSTÄTT, GERMANY 